\title{
Calcium Pyrophosphate Deposition Disease of the Temporomandibular Joint*
}

\author{
Vasisht Srinivasan, M.D. ${ }^{1}$ Andrew Wensel, M.D. ${ }^{1}$ Paul Dutcher, M.D. ${ }^{2}$ \\ Shawn Newlands, M.D., Ph.D., M.B.A. ${ }^{2}$ Mahlon Johnson, M.D., Ph.D. ${ }^{1}$ George Edward Vates, M.D., Ph.D. ${ }^{1}$ \\ ${ }^{1}$ Department of Neurosurgery; \\ 2 Department of Otolaryngology, University of Rochester Medical \\ Center, Rochester, New York. \\ Address for correspondence and reprint requests Vasisht Srinivasan, \\ M.D., Resident Physician, Department of Neurosurgery, University of \\ Rochester Medical Center, 601 Elmwood Avenue, Box 670, Rochester, \\ NY 14642 (e-mail: vasisht_srinivasan@urmc.rochester.edu).
}

J Neurol Surg Rep 2012;73:6-8.

\begin{abstract}
Keywords

- temporomandibular joint

- calcium pyrophosphate

- CPDD

- chondrocalcinosis

- tophaceous pseudogout

Calcium pyrophosphate dihydrate deposition disease (CPDD, tophaceous pseudogout) is a rare crystal arthropathy characterized by calcium pyrophosphate crystal deposition in joint spaces, episodes of synovitis, and radiological features of chondrocalcinosis. We present a case of 61-year-old woman who presented with left temporomandibular joint (TMJ) pain, difficulty chewing, left facial numbness, left-sided hearing loss, and left TMJ swelling. Imaging of the temporal fossa revealed a large mass emanating from the temporal bone at the TMJ, extending into the greater wing of the sphenoid and involving the mastoid bone and air cells posteriorly. Fine needle aspiration demonstrated polarizable crystals with giant cells. Intraoperatively, the TMJ was completely eroded by the mass. Final pathology was consistent with tophaceous pseudogout. CPDD has rarely been reported involving the skull base. None of the cases originally described by McCarty had TMJ pseudogout. Symptoms are generally pain, swelling, and hearing loss. Management is nearly always surgical with many patients achieving symptomatic relief with resection. CPDD is associated with many medical problems (including renal failure, gout, and hyperparathyroidism), but our patient had none of these risk factors. This case demonstrates that CPDD can involve the skull base and is best treated with skull base surgical techniques.
\end{abstract}

Calcium pyrophosphate dihydrate deposition disease (CPDD) is a rare crystal arthropathy characterized by the deposition of calcium pyrophosphate crystals in joint spaces, episodes of synovitis, and radiological features of chondrocalcinosis. ${ }^{1,2}$ While the disease is most prevalent in the shoulders, pelvis, knees, and joints of the hands, the axial skeleton can be affected, but rarely involves the skull base. ${ }^{2-4}$ When involved, CPDD can cause symptoms mimicking more common neurological and neurosurgical pathologies. ${ }^{2,5}$ We present a patient who was recently treated at our institution whose pathology demonstrated CPDD.

*This article was originally published online in Skull Base Reports on November 21, 2011 (DOI: 10.1055/s-0031-1296033)

\section{Case Report}

The patient is a 51-year-old right-handed Caucasian woman with a 12-month history of left ear pain that originated from the temporomandibular joint (TMJ), with some associated swelling, pain with chewing, and mild hearing loss. Her physical examination revealed mild hearing loss on the left side. Computed tomography (CT) and magnetic resonance imaging of the temporal fossa revealed a large mass emanating from the temporal bone at the TMJ (-Fig. 1A, 1B), extending into the greater wing of the sphenoid, and involving the mastoid bone and air cells posteriorly. There was erosion of the petrous carotid canal. A CT-guided biopsy was performed which diagnosed the mass as tophaceous pseudogout (tumoral CPDD).
Copyright @ $\odot 2012$ by Thieme Medical Publishers, Inc., 333 Seventh Avenue, New York, NY 10001, USA. Tel: +1(212) 584-4662.
DOI http://dx.doi.org/ 10.1055/s-0032-1329190. ISSN 2157-6971. 

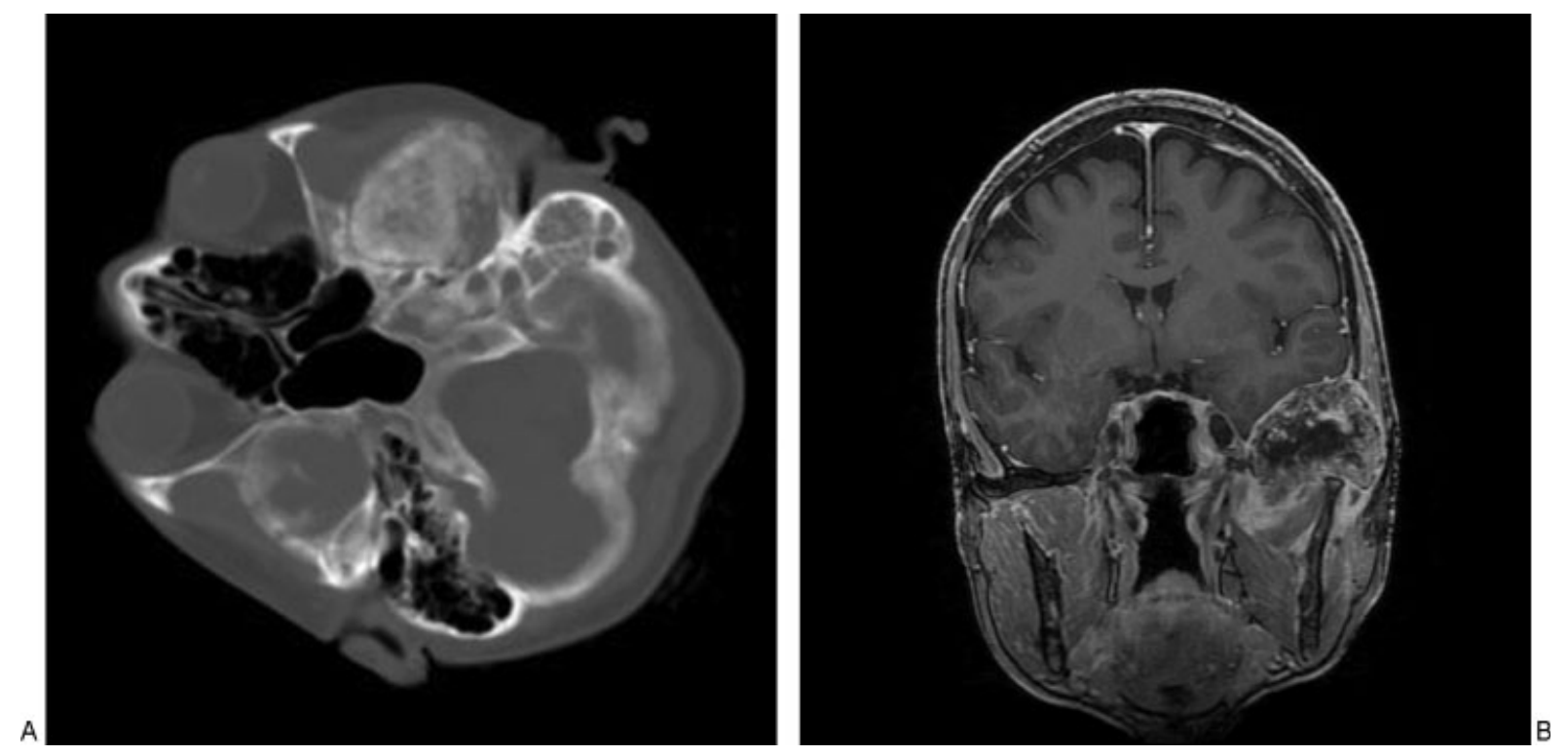

Figure 1 (A) Axial computed tomography scan demonstrating left temporomandibular joint (TMJ) erosion by a calcified lesion. (B) Coronal postcontrast T1-weighted magnetic resonance imaging demonstrating erosive lesion at the left TMJ.

Intraoperatively, the TMJ was completely eroded by the tumor. BrainLAB (Munich, Germany) was used to identify the tumor margins allowing for near gross total resection; a small portion of the lesion which was densely adherent to the internal carotid artery in the petrous canal was left behind. Initial frozen pathology results revealed "collagen with amorphous material and chronic inflammation." Final pathology demonstrated numerous polarizable, rhabdoid, and rectangular crystals, consistent with tophaceous pseudogout (-Fig. 2A, 2B).

Postoperatively, the patient did develop a cerebrospinal fluid leak and was treated with antibiotics and a lumbar drain. She was discharged 6 days after surgery with no leak. On her follow-up visits, she felt a vast improvement in her symptoms with some initial hearing loss which improved on further visits.

\section{Discussion}

CPDD arthropathy was first described in 1962 by McCarty as pseudogout due to similarity of the acute episodes of arthropathy to gout. ${ }^{2,6,7}$ Previously work had been done by Zitnan and Sitaj who had described a disorder called "chondrocalcinosis polyarticularis."2 Abnormal deposition of pyrophosphate in the joint space combines with calcium to form calcium pyrophosphate dihydrate crystals on collagen fibers; release of these crystals into the joint space results in neutrophil and monocyte-macrophage phagocytosis and release of inflammatory mediators, causing joint destruction. ${ }^{2,8}$ This becomes evident on radiological examinations as peri- and intra-articular calcifications known as chondrocalcinosis. ${ }^{9}$

CPDD arthropathy is often associated with other medical conditions, including hyperparathyroidism, hemochromatosis,
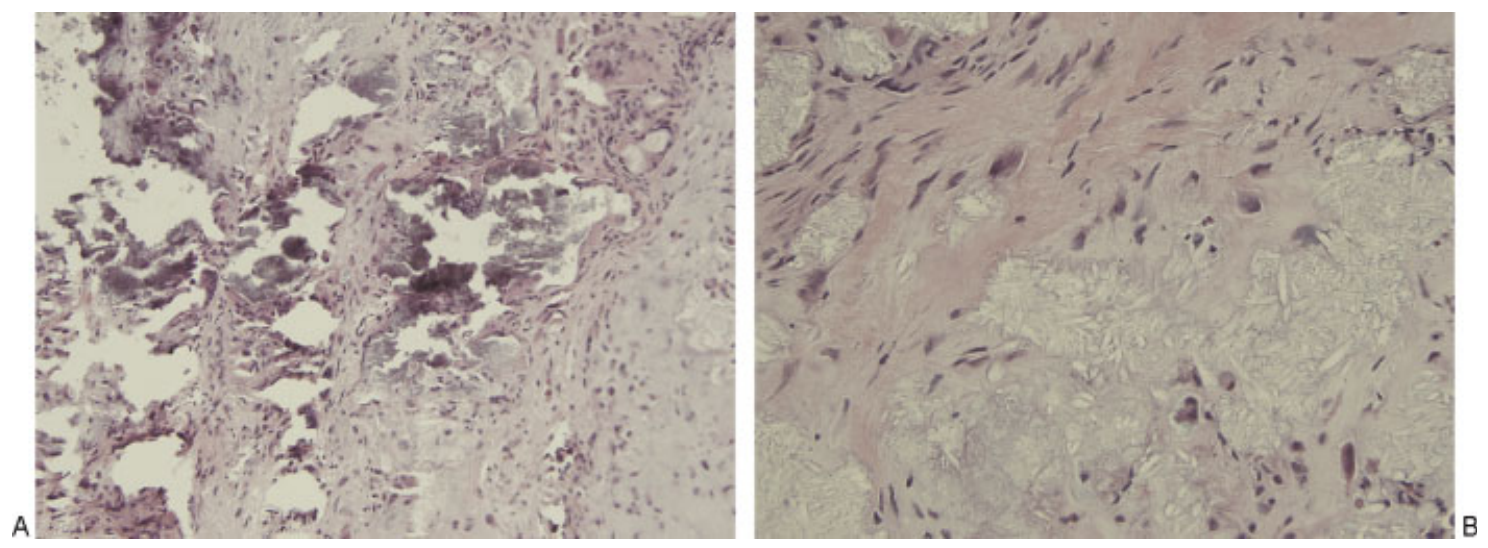

Figure 2 (A) Myxoid material with calcifications and reactive changes (H\&E: original magnification 200×). (B) Myxoid material with numerous rhomboid elongated crystals (H\&E: original magnification $400 \times$ ). 
chronic gout, renal failure, hypomagnesemia, and hypophosphatemia. ${ }^{2}$ However, our patient had none of these conditions. It may also occur on a hereditary basis but is usually sporadic; our patient has no family history of CPDD. The prevalence increases with age, occurring in some form in 10 to $15 \%$ of 65 to 75 year olds and 30 to $50 \%$ of those $>85$ years old. ${ }^{9,10}$ The disease appears to favor women $2: 1^{8,10}$ but a clear mechanism for this disparity has not been discovered. The joints most commonly affected are the knee, shoulder, hip, elbow, and metacarpophalangeal joints, while axial skeleton involvement (both cranial and spinal) is less common, but reported in the literature. ${ }^{1-3,5,9-14}$ The origin is unknown but in general, destruction of the joint is required for crystal deposition. ${ }^{2}$ Damage with increasing age predisposes to deposition due to changes in joint physiology (phosphate turnover, changes in synovial fluid components). ${ }^{2}$ However, reports also exist of postsurgical CPDD. ${ }^{13}$

CPDD involvement of the skull specifically has also been reported usually causing severe bony destruction. ${ }^{1,9,10,12,14}$ Involvement of the TMJ has been described in the literature in the form of case reports ${ }^{10,14,15}$ but was not described in any of the cases originally described by McCarty. ${ }^{7,12}$ Disease involving the TMJ typically presents with pain, swelling, and hearing loss. ${ }^{9,14,15}$ In some reports, tumoral calcinosis has been documented but this is generally in the form of hydroxyapatite crystals. ${ }^{1,2,15}$

Management of tophaceous pseudogout is surgical. While some cases have been managed conservatively, symptomatic relief in most published reports occurred only after resection of the lesion. ${ }^{14,15}$

\section{Conclusion}

CPDD can be a rare cause of common symptoms and a high threshold of suspicion is necessary to arrive at the correct etiology. As CPDD often presents in many chronic medical illnesses, neurosurgeons may be the first to diagnose these medical problems by accurately diagnosing a skull base mass as CPDD.

\section{References}

1 Grant GA, Wener MH, Yaziji H, et al. Destructive tophaceous calcium hydroxyapatite tumor of the infratemporal fossa. Case report and review of the literature. J Neurosurg 1999;90(1):148-152

2 Steinbach LS, Resnick D. Calcium pyrophosphate dihydrate crystal deposition disease revisited. Radiology 1996;200(1):1-9

3 Lam HY, Cheung KY, Law SW, Fung KY. Crystal arthropathy of the lumbar spine: a report of 4 cases. J Orthop Surg (Hong Kong) 2007;15(1):94-101

4 Salcman M, Khan A, Symonds DA. Calcium pyrophosphate arthropathy of the spine: case report and review of the literature. Neurosurgery 1994;34(5):915-918, discussion 918

5 Fenoy AJ, Menezes AH, Donovan KA, Kralik SF. Calcium pyrophosphate dihydrate crystal deposition in the craniovertebral junction. J Neurosurg Spine 2008;8(1):22-29

6 Kohn NN, Hughes RE, McCarty DJ Jr, Faires JS. The significance of calcium phosphate crystals in the synovial fluid of arthritic patients: the "pseudogout syndrome". II. Identification of crystals. Ann Intern Med 1962;56;738-745

7 McCarty DJ, Hollander JL. Identification of urate crystals in gouty synovial fluid. Ann Intern Med 1961;54;452-460

8 Molloy ES, McCarthy GM. Calcium crystal deposition diseases: update on pathogenesis and manifestations. Rheum Dis Clin North Am 2006;32(2):383-400, vii

9 Marsot-Dupuch K, Smoker WRK, Gentry LR, Cooper KA. Massive calcium pyrophosphate dihydrate crystal deposition disease: a cause of pain of the temporomandibular joint. AJNR Am J Neuroradiol 2004;25(5):876-879

10 Reynolds JL, Matthew IR, Chalmers A. Tophaceous calcium pyrophosphate dihydrate deposition disease of the temporomandibular joint. J Rheumatol 2008;35(4):717-721

11 Eichhorn GR, Berka B, Garnhaft D, Capellmann T. Lumbar radiculopathy due to chondrocalcinosis. Neurology 2006;66(10):1567

12 Mogi G, Kuga M, Kawauchi H. Chondrocalcinosis of the temporomandibular joint. Calcium pyrophosphate dihydrate deposition disease. Arch Otolaryngol Head Neck Surg 1987;113(10):1117-1119

13 Scavarda D, Litre CF, Froelich S, Srour R, Rousseaux P. Cervical tumoral calcium pyrophosphate dihydrate deposition disease 28 years after suboccipital craniotomy: case report. Neurosurgery 2007;60(6):E1151-, discussion E1151

14 Aoyama S, Kino K, Amagasa T, Kayano T, Ichinose S, Kimijima Y. Differential diagnosis of calcium pyrophosphate dihydrate deposition of the temporomandibular joint. Br J Oral Maxillofac Surg 2000;38(5):550-553

15 Smolka W, Eggensperger N, Stauffer-Brauch EJ, Brekenfeld C, Iizuka T. Calcium pyrophosphate dihydrate crystal deposition disease of the temporomandibular joint. Oral Dis 2005;11(2):104-108 